Buivys, M. and Azzopardi, L. (2016) Pienapple search: an integrated search interface to support finding, refinding and sharing. Proceedings of the Association for Information Science and Technology, 53(1), pp. 1-5. (doi:10.1002/pra2.2016.14505301122)

There may be differences between this version and the published version. You are advised to consult the publisher's version if you wish to cite from it.

This is the peer-reviewed version of the following article: Buivys, M. and Azzopardi, L. (2016) Pienapple search: an integrated search interface to support finding, refinding and sharing. Proceedings of the Association for Information Science and Technology, 53(1), pp. 1-5, which has been published in final form at 10.1002/pra2.2016.14505301122.

This article may be used for non-commercial purposes in accordance with Wiley Terms and Conditions for Self-Archiving.

http://eprints.gla.ac.uk/152410/

Deposited on: 09 January 2018 


\section{Pienapple Search: an integrated search interface to support finding, refinding and sharing}

\author{
Martynas Buivys \\ University of Glasgow \\ Glasgow, United Kingdom \\ martynas.buivyas@gmail.com
}

\author{
Leif Azzopardi \\ University of Strathclyde \\ Glasgow, United Kingdom \\ leifos@acm.org
}

\begin{abstract}
Pienapple is a search interface that aims to combine bookmarking and searching within a blended experience to facilitate improved access, serendipity, and sharing. While personal and social bookmarking platforms already exist, they are often separated from the search system, resulting in an increased effort and complexity because two or more systems need to be used. Instead, Pienapple attempts to lower the overall effort of bookmarking, (re)accessing and sharing by bringing these activities together to provide a more supportive search interface.
\end{abstract}

\section{Author Keywords}

Interactive Information Retrieval, Re-finding, Collaborative Search, Social Search

\section{INTRODUCTION}

The World Wide Web contains a vast amount of information, where typically people use search engines to discover and surface content. Once found people employ a variety of tools and employ various strategies to re-find the information discovered, either by re-querying or saving the information (or a link to) for later use and re-visitation (Sellen et al., 2002; Jhaveri, 2004; Jones et al., 2001; Wen, 2003; Obendorf et al., 2007; Aula et al., 2005). Re-finding using a search engine and the use of bookmarks are among the most common re-visitation techniques (Aula et al., 2005; Jhaveri, 2004; Obendorf et al., 2007; Jones et al., 2001). Bookmarks, also known as favourites or hotlists, were invented more than two decades ago and were used as internet shortcuts in browsers like Internet Explorer, Mozilla Firefox, and Mosaic. Over the years, bookmarks have evolved into portable collections and social sharing platforms which allow remote access and management over the internet through the use of websites and browser extensions.

Now bookmarks and search are coming together, though, there is much scope for improvements and developments in this area. For instance, Google Chrome Bookmark Manager is a browser extension for organising bookmarks. However, this bookmarking system is akin to the one provided by browsers, and so users have to go through the process of creating and organising newly added bookmarks. Furthermore, although the extension supports bookmark search it is not integrated into the standard web search interface, nor does it provide access to social bookmarks. On the other hand, Delicious, for example, is a popular social bookmarking tool that helps "...manage information traditionally kept on personal machines, while allowing for sharing with the community at large" (Lee, 2006). Although Delicious is portable and supports social exploration, bookmark search is isolated from web search. This separation requires the user to put in extra effort to visit the Delicious website in order to access and manage his/her bookmark collection. So rather than have a bookmarking tool and a search tool, we explore the possibility of making bookmarking activities, such as saving, re-finding and sharing, part of the web search experience. In this demonstration paper, we present Pienapple Search, a tool that integrates searching, bookmarking and sharing into one interface in a seamless manner. Our goal is to develop an interface that:

1. minimises the effort of bookmarking and re-finding

2. supports personal information management

3. enables collaborative and social search

In the remainder of this paper, we describe prior tools and efforts regarding personal information access, re-finding and bookmarking to provide the context and background for our novel interface. Then we outline the Pienapple Search interface and the design rationale along with the findings from a usability study involving 26 participants.

\section{BACKGROUND}

The motivation for Pienapple stems from the research on refinding and re-visitation behaviours. Re-visitations (or refinding) is a very common activity when browsing the Web. Previous studies (Tauscher \& Greenberg, 1997; Obendorf et al., 2007) indicated that around half of website visits are actually re-visits. Obendorf et al. (2007) classified re-visitation into three categories: short-term, medium-term, and long-term. While short-term re-visitations are typically supported by the browser's back button or copy-and-paste (Tauscher \& Greenberg, 1997), medium-term and long-term re-visitations often involve a variety of approaches. Jones et al. (2001) observed and interviewed eleven information specialists and researchers to understand better what methods and tools people employ to manage useful information found on the Web. Techniques 
such as emailing the URL of interest, printing or saving the web page, entering the URL directly and re-finding using a search service were among the re-access strategies employed by their subjects. Interestingly, the built-in browser tools such as bookmarks and the browsing history were not frequently used. These findings are consistent with the survey performed by Aula et al. (2005), who also noted that bookmarks are "...commonly used, but their frequency of use varies a lot". Further, they pointed out that people use a variety of strategies to re-access information and that existing tools fail to adequately support users. When using search engines to re-find information, they found that searchers find it "...difficult to remember the query terms used when finding the information in the first place" (Aula et al., 2005). Teevan, Adar, Jones, and Potts (2006) analysed the search behaviour of 114 users over 365 days using the Yahoo! search engine. By monitoring users' queries and clicks, it was discovered that "...forty percent of all observed queries $(5216 / 13,060)$ led to a click on a result that was also clicked during another query session by the same user". These findings were confirmed in the follow-up paper (Teevan et al., 2007), and the authors concluded there is a need for a tool that can effectively support finding and re-finding activities. Consequently, there has been a number of attempts to develop a variety of tools to help users.

Jhaveri and Räihä (2005) developed Session Highlights to support users as they search - letting them drag and drop URLs to a separate workspace. Thumbnails of the pages were stored in the workspace so that users could re-access previously visited pages (and thus alleviate the medium-term re-visitation problem). M. R. Morris and Horvitz (2007a) developed $S 3$, which was aimed at supporting the resumption of paused search activities (i.e. searching over multiple sessions). This prototype stored both the queries issued and pages the user marked as useful. This prototype served as the basis for SearchTogether (M. R. Morris \& Horvitz, 2007b), which aims to support collaborative web search by sharing the queries and pages found. The interface was designed to support query awareness, division of labour and persistent representation of search among small groups of people working on the same task. It also included an instant messaging system and result recommendation to enable communication between participants. More closely related to our demonstration, is SearchBar, which was developed by D. Morris, Morris, and Venolia (2008). SearchBar is a search-focused browsing history tool, which was suggested by Obendorf et al. (2007). Its goal is to improve the user experience of resuming suspended search activities and re-finding previously encountered results. It aims to assist users in regaining the context of multi-session investigations. The SearchBar implicitly registers all user's search queries and results visited and categorises them by the search topic which has to be manually created by the user. However, this introduced the problem of having to manage all the recorded queries and history. The authors try to address this problem by using a hierarchical display and rating-based filtering. In our system, we do not implicitly save and store queries and results (though that would be possible). Instead, we focus on integrating explicit bookmarking activity into the search experience. Of course, there have been numerous efforts associated specifically with bookmarking.
Delicious ${ }^{1}$ is a social computing website which allows users to store, annotate, manage and share links to useful resources on the web (Marlow et al., 2006). A Delicious bookmark contains information about the owner, the URL and the title of the resource and an optional description. All bookmarks are public by default; however users have the option to mark a bookmark as private and opt out from participating in the community. Saved bookmarks are organised by tags which are manually chosen by the user. Tags are typically only useful for the creator of the bookmark and not others. Wash and Rader (2007) interviewed twelve regular users of Delicious and discovered that tags do not help during information-seeking activities and established that "...in social discovery, it is the user and not the topic that is of interest". There are many other online bookmarking services similar to Delicious. For example, Diigo ${ }^{2}$ and Pinboard ${ }^{3}$ are two popular alternatives. Diigo enables users to bookmark and organise their online collections, as well as capture and annotate on-page elements. Similarly, Pinboard provides quick bookmarking and full-text search on bookmarks. Kippt ${ }^{4}$ was a collaborative bookmarking application which was implemented as a browser extension. It allowed the user and his/her collaborators to archive various on-page elements such as videos, articles, and images. Each saved item could be searched for, shared and discussed with collaborators. Pocket ${ }^{5}$ is another variant, which allows users to save articles they would like to read in the future. Thus, the application aims to support short-term re-visitation. However, these application did not integrate bookmarking into the user's everyday search activities.

On the other hand, browsers provide support for bookmarking and re-finding. For example, the Google Chrome Bookmark Manager is an extension for the Google Chrome browser which stores the user's bookmarks in the cloud so they can be accessed anywhere using any device. The process of bookmarking a page is similar to using a browser's bookmarking functionality. The user needs to select where the bookmark is saved too, give the bookmark a name (if they like), enter the name of the folder it is to be saved to, etc. Bookmarks are presented as big thumbnails to provide visual cues. The application allows you to search your bookmark collection easily using the URL bar and quickly re-find saved information. Other browsers such as Edge, Firefox and Safari also provide similar support.

As outlined above, there has been a variety of different bookmarking applications. However, we posit, that because they are not fully integrated with how or when bookmarks are actually used and when they are useful - this limits the utility of bookmaking. Said another way, the effort involved in booking and re-finding through a separate interface typically outweighs the benefit of doing so, especially when it is possible to requery for the information. Our aim in developing Pienapple is to reduce the effort required, and provide more seamless access through an integrated search interface.

\footnotetext{
1 delicious .com

2 www.diigo.com

3 pinboard.in

4 kippt. com

5 getpocket.com
} 


\begin{tabular}{l|l} 
p̈ienopple & python \\
Bookmarks Web Images News Videos & \\
\hline
\end{tabular}

Python Data Analysis Library - pandas: Python Data Anal...
http://pandas.pydata.org/
\& martynasb $\square$ Machine Learning

4.7 Slicing Arrays

http://structure.usc.edu/numarray/node26.html

o martynasb $\square$ Python

Overview - Python 3.5.1 documentation

https://docs.python.org/

Python 3.5.1 documentation. Welcome! This is the documentation for Python 3.5.1, last updated May 07, 2016. Parts of the documentation:

\& martynasb $\square$ Python

Learn Python - Free Interactive Python Tutorial

http://www.learnpython.org/

LearnPython.org is a free interactive Python tutorial for people who want to learn

Python, fast.

S leifos $\square$ python

The Python Tutorial - Python 2.7.11 documentation https://docs.python.org/2/tutorial/

The Python Tutorialף Python is an easy to learn, powerful programming language. It has efficient high-level data structures and a simple but effective approach to ...

\& leifos $\square$ python

\section{SUGGESTED FOLDERS 0 \\ $\square$ Machine Learning \\ $\checkmark$ Python}

BOOKMARK FOLDERS 0

$\square$ Personal
$\square$ Read later
$\square$ Work
$\square$ Machine Learning
$\square$ University
$\square$ Research
$\square$ JavaScript
$\square$ Java
$\square$ Web development
$\square$ Design
$\square$ Python
$\square$ Movies
$\square$ Tools
$\square$ Programming
$\square$ Pienapple
$\square$ Python ide
$\boxplus$ Create a new folder

Figure 1. Pienapple Search interface (WWW. pienapple. com). When the user starts typing a query (i.e. "python"), results from their own bookmarks are recommended (personal space), along with recommendations from other users (social space). Folders relevant to the query are also recommended in the top right. If the user hits return or pressed the search button, then a web search is conducted (see Figure 2).

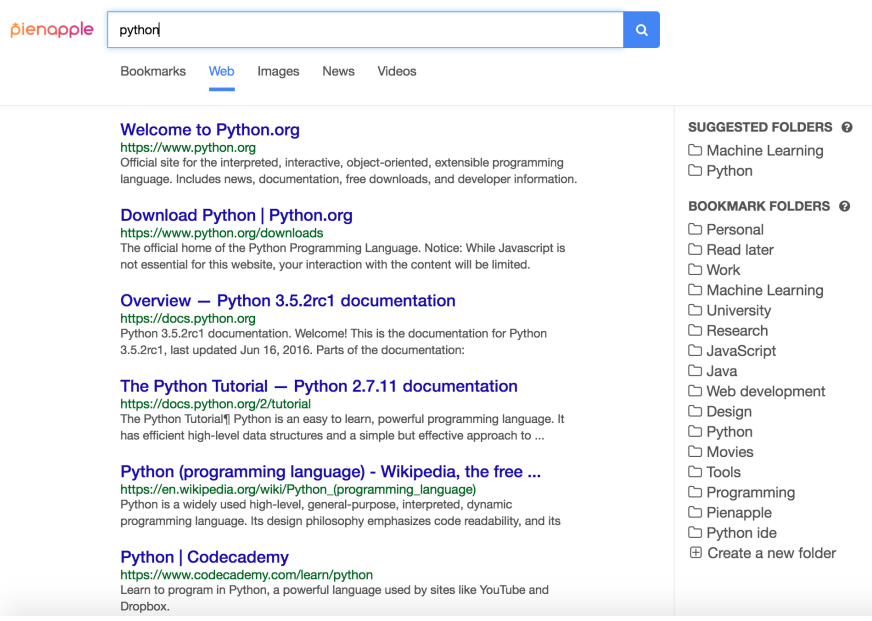

Figure 2. Pienapple Search interface (wWW. pienapple . com) on the web vertical for the query "python". The user can drag and drop results to folders, or visit them as usual, and use the browser extension to bookmark pages into their Pienapple folders.

\section{PIENAPPLE SEARCH}

Pienapple Search aims to integrate bookmark search and management within a typical web search interface. The system is deployed in the public domain, and it can be accessed by visiting WwW. pienapple.com. The interface was designed to: (i) minimise changes to the standard web search interface, (ii) minimise the effort of the user's bookmarking actions, and (iii) minimise the amount of effort involved in re-finding. An additional aim was to facilitate social exploration and sharing of bookmarks. Therefore, the Pienapple Search interface is similar to traditional web search interfaces: a query box, ten blue links of results and tabs to display the verticals (i.e. web, images, news and videos), see Figure 2. The look and feel are consistent with the minimalism of search engines such as Google and Bing. The modifications to the interface are as follows: (i) a bookmarks vertical, (ii) a list of bookmark folders (on the right), and (iii) snippets on the bookmarks vertical also include who "owns" the bookmark, i.e. Pienapple username and folder name are shown (see Figure 3).

Searching: The mechanics of searching are as follows. As the user types their query, Pienapple tries to match the partial query to the user's current bookmarks and recommends any matching bookmarks. If the user continues typing and then 
hits search, the request is then submitted to the web vertical, and the web results are returned. This seamlessly integrates bookmark search and web search together.

Bookmarking: To do this, we first made the bookmark folders visible on the search interface and provided instant access to bookmarks by providing an additional tab (bookmarks). To facilitate access to bookmarks, as the user enters a querying, relevant bookmarks are displayed, until the user hits return or the search button, in which case a web search is performed. To reduce the effort of bookmarking, we provide a number of features: the ability to drag and drop results to folders from within the search interface, or if on a web page, the user can click the Pienapple extension to bookmark it. To further reduce the cost, as the user types a query we automatically suggest folders that are likely to be relevant to the query and suggest the creation of new folders based on the query.

Folder suggestion happens at two levels: (i) suggesting existing folders and (ii) suggesting new folders. For example, if the user had folders about programming, i.e. "python", "perl", etc., and the query was "programming", then these folders would be recommended. If they added "python" to the query, then only the python folder would be suggested. In the second case, the interface would also suggest the create of a new folder, "python programming" as another possible folder to store bookmarks. The idea with these suggestions is again to minimise the effort of folder creation and bookmark organisation. Currently, two folders are recommended.

Sharing: To share bookmarks, a user needs to mark their folder as public. Then the list of bookmarks can be accessed by visiting: wWw.pienapple.com/<username $>/<$ folder -name $>$. Sharing also happens implicitly. When searching on the bookmark vertical, bookmarks saved by other users are also recommended. These "social" bookmarks are indicated by including the username of the owner and the folder name. The idea here is that by showing such links to users will enable users to explore bookmarks others have found. Currently, we have only implemented public and private options. So it would be interesting to explore how such an option could be integrated with social networks to constrain where or which bookmarks are recommended.

\section{How to Tango with Django: A Python Django Tutorial http://www.tangowithdjango.com/ \\ Tango With Django 1.7. A beginner's guide to web development with Python 2.7 / Django 1.7. Comments, suggestions and feedback is welcome. Changes requests $\checkmark$ leifos $\square$ django}

Figure 3. Bookmark Snippet includes the owner (leifos) and folder name (django). These can be selected by the user to find other related bookmarks by the owner and in the folder.

\section{Implementation}

Pienapple Search project is implemented as a componentbased system, consisting of seven independent services ${ }^{6}$ : Web Client, an API service, MongoDB database, ElasticSearch

\footnotetext{
6wW.mongodb.org, wWw.elastic.co, redis.io, wWw.alchemyapi.com, datamarket . azure.com/dataset/bing/search
}

bookmark search service, Redis queue, Alchemy API services, Microsoft Bing search service.

The Web Client is the user interface the user interacts with to search the web and manage the bookmarks. It is built using the React JS framework and utilises the Facebook's Flux architecture. The Client is an independent component that communicates with the API through a RESTful interface by sending and receiving JSON payload. In addition, the Client is responsible for logging all user's actions. The advantage of using a client-side logger over a server side logger is that it can capture richer data about how a user interacts with the interface.

The Pienapple API provides a public interface for clients to access the MongoDB database and the ElasticSearch bookmark search service. It also orchestrates the Redis job queue for background workers responsible for bookmark indexing. The API is implemented using Node JS language and runs on Express framework. It is plugged into Travis CI, a continuous integration framework, which automatically runs a set of tests against all API endpoints to ensure the newly committed code does not break the application. The ElasticSearch bookmark search service is responsible for retrieving bookmarks based on a given search query. The client does not have direct access to the search service. Instead all requests by the client are proxied through the Pienapple API. Similarly, the Microsoft Bing search service is responsible for supporting searches in four different verticals: web, news, images, and videos. The API delegates the Client's search requests in these verticals to Microsoft Bing Search API and returns the results in JSON format. Redis queue is used by the API for background jobs. For example, when a Pienapple Search user creates a bookmark, a few background jobs will be created. When a background worker becomes available, a request will be sent to the Alchemy API service to extract the content of the bookmarked page. After that, the bookmark will be indexed by ElasticSearch search service. Alchemy API is used by the background workers to extract the text and named entities from bookmarked pages. The extracted content is then pushed to the ElasticSearch bookmark search service to improve the quality of the search results.

\section{USABILITY STUDY}

Given the Pienapple interface described above we conducted a usability study to determine what areas could be improved and to receive feedbacks on the application. At our university, we recruited 28 master's students to perform a task based usability study. The participants were briefly introduced to the interface and then asked to perform a simulated leisure task, where participants had to imagine that they were hosting an Italian night for their friends. They were asked to find, bookmark, and then later re-find pizza recipes that would appeal to their friends. Participants answered a number 5point Likert questionnaire about features of the interface (see Table 1), a standard 10-item System Usability Scale (SUS) questionnaire (Brooke, 1996) for measuring the perceived system usability, and three open-ended questions.

From the initial questions, participants felt that the interface integrates bookmarks well and provides good recommenda- 
Table 1. Likert Questions on Pienapple Usability (1strongly disagree to 5-strongly agree).

\begin{tabular}{ll}
\hline Question & Mean (Std) \\
\hline Good Integration & $4.27(0.92)$ \\
Good Recommendations & $4.12(0.91)$ \\
Easy to Manage & $4.23(0.91)$ \\
Preferred over other bookmarking tools & $4.04(0.82)$ \\
Preferred over other search engines & $3.23(1.11)$ \\
Easy to Use & $3.58(1.14)$ \\
\hline
\end{tabular}

tions (i.e. suggesting folders into which URLs can be saved). They also felt it was it was relatively easy to manage their bookmarks. Participants felt it was a better bookmarking tool that other tools that they had previously used, but were mixed about whether it was better than existing search engines. There was also less agreement regarding whether it was easy to use. This was re-confirmed by the SUS questionnaire results where the interface was rated as 77 out of 100, which is considered to be good as the minimum usability score is considered to be 68 (Brooke, 1996). However, of the 28 participants, five rated the system below 60 , with the lowest being 30 . So this suggests that there is much room for improvement.

We asked three open-ended questions regarding what they most liked, least liked, and what they would improve in the system. We do not have space to detail all the points, and so below we provide a summary of the feedback. Re-affirming the Likert questions participants mentioned that liked the bookmark creation $(N=4)$, organisation $(N=6)$, and search $(N=4)$. In addition, the participants liked the drag and drop bookmarking $(N=3)$ and the Google Chrome extension to quickly bookmark the current page $(N=2)$. For the least favourite features of the system, participants listed the limited sharing capability (i.e. currently a web link to the bookmarks as oppose to via social media) $(N=2)$ and no obvious delineation between the personal bookmark search results and the results from other users. The participants also provided some suggestions on how to improve the interface. These included: nested folder support $(N=3)$, the ability to re-arrange bookmarks folders $(N=2)$, more intuitive or explicit ways to bookmark, as dragging and dropping was not obvious to some participants, and improved folder permissions (e.g. making it more obvious that folders can be made private or public) and highlighting whether a bookmark is personal or social. The suggestions by participants indicated more work is needed to make the experience more seamless and the interface more intuitive.

\section{SUMMARY AND FUTURE WORK}

In this demonstration paper, we have presented Pienapple Search, which aims to combine personal, social and web search together in a blended experience. From our usability study, while generally quite positive, we have identified a number of issues that need to be addressed before deploying the system and undertaking task-based evaluations and naturalistic studies. Once deployed we will focus our attention on more backend IR problems: (i) bookmark folder suggestion (ii) bookmark ranking and (iii) sharing and suggesting bookmarks to other users.

\section{References}

Aula, A., Jhaveri, N., \& Käki, M. (2005). Information search and re-access strategies of experienced web users. In Proc. of the 14th www conference (pp. 583-592).

Brooke, J. (1996). Sus: A quick and dirty usability scale.

Jhaveri, N. (2004). Intermediate and post-session web page revisitation techniques and tools (Unpublished master's thesis). Department of Computer Sciences, University of Tampere, Finland.

Jhaveri, N., \& Räihä, K.-J. (2005). The advantages of a crosssession web workspace. In Chi '05 extended abstracts (pp. 1949-1952).

Jones, W., Bruce, H., \& Dumais, S. (2001). Keeping found things found on the web. In Proc. of the 10th cikm conference (pp. 119-126).

Lee, K. J. (2006). What goes around comes around: An analysis of del.icio.us as social space. In Proc. of the 20th cscw conference (pp. 191-194).

Marlow, C., Naaman, M., Boyd, D., \& Davis, M. (2006). Ht06, tagging paper, taxonomy, flickr, academic article, to read. In Proc, of the 17th hypertext conference (pp. $31-40)$.

Morris, D., Morris, M. R., \& Venolia, G. (2008). Searchbar: A search-centric web history for task resumption and information re-finding. In Proc of the acm chi conference (pp. 1207-1216).

Morris, M. R., \& Horvitz, E. (2007a). S3: Storable, shareable search. In Interact (p. 120-123).

Morris, M. R., \& Horvitz, E. (2007b). Searchtogether: An interface for collaborative web search. In Proc. of the 20th acm uist symposium (pp. 3-12).

Obendorf, H., Weinreich, H., Herder, E., \& Mayer, M. (2007). Web page revisitation revisited: Implications of a longterm click-stream study of browser usage. In Proc. of the acm chi conference (pp. 597-606).

Sellen, A. J., Murphy, R., \& Shaw, K. L. (2002). How knowledge workers use the web. In Proc. of the acm chi conference (pp. 227-234).

Tauscher, L., \& Greenberg, S. (1997). Revisitation patterns in world wide web navigation. In Proc. of the acm chi conference (pp. 399-406).

Teevan, J., Adar, E., Jones, R., \& Potts, M. (2006). History repeats itself: Repeat queries in yahoo's logs. In Proc. of the 29th acm sigir conference (pp. 703-704).

Teevan, J., Adar, E., Jones, R., \& Potts, M. A. S. (2007). Information re-retrieval: Repeat queries in yahoo's logs. In Proc. of the 30th acm sigir conf. (pp. 151-158).

Wash, R., \& Rader, E. (2007). Public bookmarks and private benefits: An analysis of incentives in social computing. Proc. of the ASIST, 44(1), 1-13.

Wen, J. (2003). Post-valued recall web pages: User disorientation hits the big time. IT \& Society, 1, 184-194. 